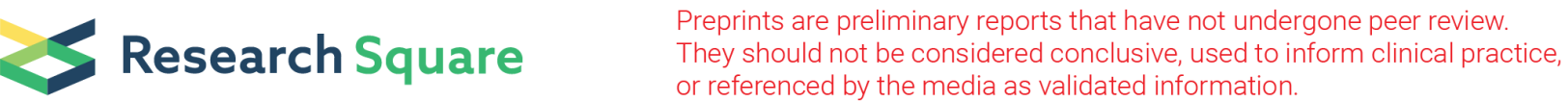

\section{Sensory Processing in Children and Adolescents Shortly After the Onset of Anorexia Nervosa: A Pilot Study}

\section{Tasuku Kitajima ( $\square$ tkita@dokkyomed.ac.jp )}

Dokkyo Medical University Saitama Medical Center: Dokkyo Ika Daigaku Saitama Iryo Center https://orcid.org/0000-0003-3333-7139

\section{Ryoko Otani}

Dokkyo Medical University Saitama Medical Center: Dokkyo Ika Daigaku Saitama Iryo Center

\section{Takeshi Inoue}

Dokkyo Medical University Saitama Medical Center: Dokkyo Ika Daigaku Saitama Iryo Center

\section{Naho Matsushima}

Dokkyo Medical University Saitama Medical Center: Dokkyo Ika Daigaku Saitama Iryo Center

Naoki Matsubara

Dokkyo Medical University Saitama Medical Center: Dokkyo Ika Daigaku Saitama Iryo Center

\section{Ryoichi Sakuta}

Dokkyo Medical University Saitama Medical Center: Dokkyo Ika Daigaku Saitama Iryo Center

\section{Research Article}

Keywords: sensory profile, hypersensitivity, sensory processing, anorexia nervosa, adolescents

Posted Date: February 8th, 2022

DOl: https://doi.org/10.21203/rs.3.rs-1319329/v1

License: (c) (i) This work is licensed under a Creative Commons Attribution 4.0 International License. Read Full License 


\section{Abstract}

\section{Background}

Alterations in sensory processing, such as vision, taste, and interoceptive sensation, have been reported in adult anorexia nervosa (AN). Whether these symptoms are traits, states, or "scars" due to starvation has not been fully established. Based on the hypothesis that alterations in sensory processing also occur in adolescent AN in the early stages of the disease, the present study was conducted using both selfadministered and parent-administered sensory processing questionnaires.

\section{Methods}

Children and adolescents with anorexia nervosa treated at a single tertiary eating disorder treatment center in Japan (AN group) and female junior high school students attending a public junior high school in Saitama Prefecture, Japan (healthy control group: HC group) were included in the study. The Sensory Profile (SP) and Adult/Adolescent Sensory Profile (AASP) were administered to the participants and their caregivers. In addition, we collected demographic data and administered the Children's Eating Attitude Test and Autism-Spectrum Quotient Children's version.

\section{Results}

Seventeen children and adolescents were enrolled in the AN group, and 63 were enrolled in the HC group. There was no statistically significant difference between the $\mathrm{AN}$ and $\mathrm{HC}$ groups in the quadrant scores of the AASP. In the SP, the Sensory Avoidance score and the Emotional/Social response score were higher in the AN group than in the $\mathrm{HC}$ group.

\section{Conclusions}

Although this study was conducted at only a single center in Japan, the results suggest that sensory processing characteristics may differ from trait symptoms. Longitudinal studies and larger-scale studies with patients in the early stages of the disease are needed.

\section{Background}

Anorexia nervosa (AN) is a common psychiatric disorder that presents with a variety of psychosomatic symptoms and abnormal eating behaviors. [1] AN was believed to be caused by psychosocial factors, but in recent years, evidence has accumulated that $\mathrm{AN}$ is a disease associated with biological changes that develop due to a combination of genetic and environmental factors [2]. However, the biological pathogenesis that can explain the symptoms in a unified manner has yet to be elucidated. In addition, it is not yet possible to distinguish whether the various symptoms of AN are traits that exist before the onset of the disease, states that reflect the condition after the onset of the disease, or "scars" due to starvation. Distinguishing these symptoms is important for investigating factors that contribute to the 
development and maintenance of AN and for developing new treatments, but longitudinal studies are difficult to perform because of their cost and the high levels of attrition among individuals with AN.

Body image disturbance is one of the central symptoms of AN. Several studies have reported the presence of multisensory impairment in $\mathrm{AN}$, which has been suggested to lead to body image disturbance. [3-5]

There are several reports on the subjective sensory experience of patients with AN in which selfadministered tests were used as the method to assess sensory impairment [6-8]. It has been suggested that AN is a trait symptom regardless of its nutritional status because abnormal sensory processing is observed in AN in both the acute and remission phases [8]. If AN is a trait symptom, multisensory impairment should be observed from the early onset of AN. However, these studies included adults and adolescents more than one year after the onset of AN, and it is not clear whether the sensory processing problems existed before the onset or emerged after the onset [6-8].

To assess sensory impairment, several physiological examinations are used in addition to questionnairebased assessment [9-20]. Questionnaires are easy to administer, but they do not capture objective changes in sensory processing. Physiological examinations provide objective indicators but are often difficult to perform clinically because of the complexity of the examinations, and examinations of responses to smell and pain are often invasive for patients with AN. To develop a treatment based on the state of multisensory impairment, it is desirable to use a simpler method of assessment.

Although multisensory impairment has been investigated using subjective self-administered questionnaires[3-5], there is no objective study based on the evaluation of others, such as parents and caregivers. In recent years, it has been found that the involvement of family members in the treatment of child and adolescent AN improves the prognosis [21]. It is clinically meaningful to understand not only the subjective sensory experience of the patient but also the way in which the family views it.

In the present study, we aimed to verify the hypotheses that "abnormalities in subjective sensory experiences suggestive of multisensory impairment can be observed even in children and adolescents with early-stage AN" and "multisensory impairment in children and adolescents with AN can be accurately captured through the objective assessments of family members."

\section{Methods}

\section{Participants}

This study was conducted among children and adolescents with AN requiring inpatient treatment and their caregivers (the AN group) and age-matched healthy controls and their caregivers (the HC group).

The AN group consisted of children aged 11-18 years who first visited the Child Development and Psychosomatic Medicine Center at Dokkyo Medical University Saitama Medical Center from June 23, 
2019 , to December 31, 2020. They and their parents gave consent and assent to participate in the study. They were diagnosed according to the DSM-5, and two specialists in child psychiatry certified by the Japanese Society for Child and Adolescent Psychiatry, the Japanese Society of Pediatric Psychiatry and Neurology and the Japanese Society of Psychosomatic Pediatrics confirmed the diagnosis. All participants in the AN group were monitored for more than one year, and their diagnoses, all AN restrictive type, were confirmed to be correct.

As the $\mathrm{HC}$ group, female students who attended a public junior high school in Saitama Prefecture, Japan, and their parents or caregivers were recruited. Among the control group, participants were excluded from this study if they were found to have a psychiatric or neurodevelopmental disorder.

\section{Procedure}

The children in the AN group completed the questionnaire test battery at the time of their first outpatient visit. Demographic data were collected at the time of the test, and height and weight were extracted from the medical records as measured on the day of the test or within a week. For the control group, information on age, sex, and self-reported height and weight were collected when the questionnaire was administered. The demographic and background data are presented in Table 1.

\section{Assessment Measures}

\section{Sensory Profile (SP) and Adolescent/Adult Sensory Profile (AASP)}

The Sensory Profile is a standardized instrument for assessing sensory processing and profiling the impact of sensory processing on functional behavior in daily life [22-24]. Sensory processing patterns are classified into four quadrants (Low Registration, Sensation Seeking, Sensation Sensitivity, and Sensation Avoidance) based on high and low neurological thresholds for sensory stimuli, and the behavioral responses (passive and active) associated with those thresholds are scored for each quadrant. Low Registration indicates the degree to which a person misses sensory input, Sensation Seeking indicates the degree to which a person explores sensory input, Sensory Sensitivity indicates the degree to which a person detects sensory input, and Sensation Avoidance indicates the degree to which a person is bothered by sensory input [22] .

The Sensory Profile is an objective scale that is assessed by the parents and other caregivers [22, 24]. The SP was developed by Dunn et al., translated into Japanese, and standardized for ages 3-82, and it is widely used in clinical settings, especially in the field of occupational therapy [24,25]. The questionnaire consists of 125 items divided into three major categories: the sensory processing category (auditory, visual, vestibular, touch, multisensory, and oral sensory processing), which indicates the child's responses to the basic sensory systems; the coordination category (sensory processing related to endurance/tone, 
modulation related to body position and movement, the modulation of movement affecting activity level, the modulation of sensory input affecting emotional responses, and the modulation of visual input affecting emotional responses and activity level), which reflects participants' control of neurotransmissions from the facilitation or inhibition of various responses; and the behavior and emotional responses category (Emotional/Social responses, Behavioral outcomes to sensory processing, and Items indicating thresholds for response), which reflects the results of participants' sensory processing in their behavior $[24,25]$. Each item is scored on a five-point Likert scale. In this study, we examined the quadrant and section scores.

The AASP is a self-rated questionnaire that can be used to assess subjective sensory processing experiences. $[23,26]$ The AASP was also developed by Dunn et al. and translated into and standardized in Japanese. The AASP is a self-assessment questionnaire that can be used to assess subjective sensory processing experiences. It consists of 60 items in the following sections: Taste/Smell, Movement (Vestibular/Proprioceptive), Visual, Touch, Activity level, and Auditory processing. Each item is scored on a five-point Likert scale.

\section{Children'S Eating Attitude Test (Cheat-26)}

The ChEAT-26 is a 26-item self-administered questionnaire assessing eating attitudes and behavior [27, 28]. The child version has since been validated in Japan [29]. Each item is scored on a six-point Likert scale: "never," "rarely," "sometimes," "often," "usually," and "always." In the Japanese version, a score of 18 and above indicates that an individual should be considered for eating disorders, and the 26 items are divided into five subscales assessing "preoccupation with thinness", "food preoccupation", "dieting", "social pressure to eat", and "purging" [29].

\section{Autism-spectrum Quotient Children'S Version (Aqc)}

The AQC is a 50-item parent-administered questionnaire assessing autistic traits for children [30]. The Japanese version has already been validated for children between the ages of 6 and 15 years [31]. In the Japanese version, a score of 25 and above indicates that an individual should be considered for a specialist autism assessment, and this version is divided into five subscales assessing "social skills", "attention switching", "attention to detail", "communication" and "imagination." [31]

\section{Analysis}

All data were analyzed using the IBM SPSS version 28 (Armonk, NY: IBM Corp.). The normality of data was assessed using the Shapiro-Wilk test, and normally distributed data were tested for equivariance using the Levene test. Based on the results of the normality test, comparisons between the AN and $\mathrm{HC}$ groups were performed with unpaired $t$ tests for age, height, weight, BMI, and BMI-SDS and with the 
Mann-Whitney U test for SP/AASP, AQC, and ChEAT-26 score comparisons. Spearman's rank correlation coefficient was used for correlation analysis. For all tests, a two-tailed $p$ value $<0.05$ was considered statistically significant.

\section{Results}

The demographic and background data of the participants are shown in Table 1. There was no significant difference in age between the AN group and the control group. Weight, BMI, and BMI-SDS were all significantly lower in the AN group than in the control group, a finding consistent with the diagnosis of AN.

The AQC score tended to be higher in the AN group than the control group for the total score and subscales, but only the difference in the "attention to detail" score was statistically significant. In the ChEAT, the score of the AN group was statistically significantly higher than that of the control group on all subscales except "preoccupation with thinness" and in the overall score.

Table 2 shows the quadrant and section scores on the AASP for the AN and control groups. Contrary to our hypothesis, there was no statistically significant difference between groups in the quadrant and section scores on the AASP.

Table 3 shows the quadrant and section scores of the SP for the AN and control groups. Among the quadrant scores, only the Sensation Avoidance score was significantly higher in the AN group than the control group ( $p<0.01, r=0.35)$, while the Emotional/Social responses score tended to be significantly higher in the AN group, and Vision tended to be higher in the AN group, but the effect size was low.

As an exploratory study, we examined the correlations between AASP and SP quadrant scores and BMI, total ChEAT scores, and subscales (Table 4). In the AN group, AASP Sensory Sensitivity and Sensation Avoidance were significantly correlated with the ChEAT total score and the subscale scores of "preoccupation with thinness" and "food preoccupation". Although the HC group also showed a weak correlation with "preoccupation with thinness," the AN group showed a stronger correlation. In contrast, in SP, Sensation Seeking was correlated with the ChEAT total score and "food preoccupation," "dieting," and "social pressure to eat".

\section{Discussion}

To the best of our knowledge, this is the first study to assess the sensory processing characteristics of children and adolescents with AN in the early and acute stages, less than one year after the onset. Based on reported previous findings, we hypothesized that children with AN in the very early stages would have altered sensory processing, similar to that of adults with AN.

However, the results did not support our hypothesis, especially in the evaluation of subjective sensory experiences. There was a significant difference between groups in the objective measure, the Sensation 
Avoidance score, but no significant difference between groups in the subjective measure. In the SP, many items in Emotional/Social responses are included in Sensation Avoidance items, so higher Emotional/Social response scores tend to be associated with higher Sensation Avoidance scores. Patients with anorexia nervosa often struggle with emotion regulation and can have high scores in Emotional/Social response without necessarily having altered sensory processing. If we consider purely the sensory processing aspect, it is possible that children and adolescents at a very early stage of AN participated in our study were not experiencing alterations in sensory processing compared to HCs. The fact that the SP Sensory Sensitivity score of the AN group was not higher than that of the HC group would support this idea. Considering this, the SP score, as assessed by parents or caregivers, may not correctly reflect the sensory processing characteristics of individuals with AN.

Previous reports have shown that patients with AN show sensory hypersensitivity in questionnaires of subjective sensory processing characteristics [6-8]. In addition, sensory sensitivity is reported to be associated with weight loss. Furthermore, it has been reported that hypersensitivity persists even after weight regain $[6,7]$. However, in this study of children and adolescents with eating disorders in the early and acute stages, there were no findings of hypersensitivity compared to healthy control participants. On the SP, an objective caregiver assessment, the AN group consistently showed no significant difference in sensory sensitivity scores compared to the HC group. We want to discuss the possible reasons for this finding in terms of two points.

The first point is that the timing of the present study was in the acute phase of treatment. In a previous report that evaluated sensory processing characteristics before and after acute inpatient treatment, it was reported that overresponsiveness to sensory stimuli was higher at the time of weight gain, which may have resulted in undervaluation at the time of the present study. However, in this report, the participants were overresponsive to sensory stimuli even before they gained their weight compared to healthy participants [7]. Therefore, it is unlikely that the acute stage of evaluation was the only factor in the present results.

The second point is that most of the patients evaluated in this study were in the first episode and very early stage of the disease, less than one year after onset. Previous reports have focused on patients more than one year after the onset of symptoms and have not examined whether sensory responses differ in the early stage of the disease $[4,6-8]$. This study is the first to report on this issue. Therefore, it is possible that the changes in sensory processing have not yet appeared in patients with very early-stage AN. It is believed that the starvation associated with AN causes changes in the reward system, particularly an abnormally elevated prediction error response, which plays an important role in the learning process [3235], and adolescents with AN have higher stimulus-response learning in both implicit and explicit learning [36]. These findings lead to the hypothesis that responses to sensory stimuli change to overresponsiveness or hypersensitivity due to altered learning processes after starvation due to AN. Based on this hypothesis, hypersensitivity in AN may not be a trait symptom but rather a "scar" caused by emaciation. The present study was not designed to examine the relationship between sensory processing 
and learning process changes, so we cannot discuss this hypothesis, but the relationship between sensory processing and reward system changes can be an issue for future research.

In the present study, we also found correlations between Sensory Sensitivity, Sensation Avoidance and ChEAT-26 preoccupation with thinness and food preoccupation scores in adolescents with AN but not with BMI-SDS. This suggests that sensory sensitivity is related to the pathology of eating disorders and may also have a state-symptom component that is not related to weight. The finding that sensory processing problems have a state-symptom component is consistent with previous reports using physiological examinations [37]. To clarify whether these symptoms in patients with AN are traits, states, or "scars," larger studies with patients in the very early stages of the disease or studies with twins may be helpful [38].

It is clinically meaningful to understand the sensory processing characteristics of patients with AN. However, the SP and AASP have many items and are burdensome, making them unsuitable for longitudinal assessments. A simple method with a small number of items, such as the Brief Sensory Screener, may be used for longitudinal evaluation [8].

There are several important limitations to this study. First, the study was conducted at a single center in Japan, which may have led to selection bias. There have been no reports examining sensory processing characteristics in patients with AN in Japan. The small number of patients with AN is also an important limitation. Therefore, it is possible that sensory sensitivity in the AN group was underestimated. It is necessary to examine whether adult patients with AN in Japan show the same sensory processing abnormalities as those previously reported and to examine a larger number of child and adolescent patients.

However, the findings of the present study, which examined sensory processing characteristics in the early stages of AN, are significant in that they suggest that sensory processing characteristics may be both states and "scars" due to starvation.

\section{Conclusion}

This study examined sensory processing characteristics in children and adolescents with early-stage AN using the Sensory Profile. No significant difference in Sensory Sensitivity was found between the AN group and the $\mathrm{HC}$ group. The results suggest that researchers should reconsider that sensory processing characteristics are trait symptoms, as previously thought. However, because of the important limitations of this study, it is not clear whether alterations in sensory processing characteristics in AN patients are due to states, traits or "scars" due to undernutrition.

\section{List Of Abbreviations}

AN

Anorexia nervosa 
$\mathrm{HC}$

Healthy control

SP

Sensory Profile

AASP

Adult/Adolescent Sensory Profile

ChEAT-26

Children's Eating Attitude Test

AQC

Autism-Spectrum Quotient Children's version

BMI

Body mass index

SDS

Standard deviation score

\section{Declarations}

\section{Ethics approval and consent to participate}

This study passed the Ethics Review Committee of the Saitama Medical Center of Dokkyo Medical University (No. 1904) and was conducted with the written consent of the participants. The study was conducted in accordance with the Declaration of Helsinki.

\section{Consent for publication}

Not applicable

\section{Availability of data and materials}

The datasets analyzed in the current study are available from the corresponding author on reasonable request.

\section{Competing interests}

The authors declare that they have no competing interests.

\section{Funding}

This work was supported by JSPS KAKENHI Grant Number JP18K02797.

\section{Authors' contributions}

TK designed the study; collected, analyzed, and interpreted the data; and wrote the original draft. RO, TI, NM, and NM interpreted the results, and RS supervised the study. The authors read and approved the final 
manuscript.

\section{Acknowledgments}

Not applicable

\section{References}

1. American Psychiatric Association. Diagnostic and statistical manual of mental disorders (DSM-5®):. American Psychiatric Pub; 2013.

2. Zipfel S, Giel KE, Bulik CM, Hay P, Schmidt U. Anorexia nervosa: aetiology, assessment, and treatment. Lancet Psychiatry. 2015;2(12):1099-111.

3. Cascino G, Castellini G, Stanghellini G, Ricca V, Cassioli E, Ruzzi V, Monteleone P, Monteleone AM. The Role of the Embodiment Disturbance in Anorexia Nervosa Psychopathology: A Network Analysis Study. Brain Sci. 2019;9(10):276. doi:10.3390/brainsci9100276.

4. Gaudio S, Brooks SJ, Riva G. Nonvisual multisensory impairment of body perception in anorexia nervosa: A systematic review of neuropsychological PloS one 2014,9 (10):e110087.

5. Riva G, Dakanalis A. Altered Processing and Integration of Multisensory Bodily Representations and Signals in Eating Disorders: A Possible Path Toward the Understanding of Their Underlying Causes. Front Hum Neurosci 2018, 12.

6. Zucker NL, Merwin RM, Bulik CM, Moskovich A, Wildes JE, Groh J. Subjective experience of sensation in anorexia nervosa. Behav Res Ther. 2013;51(6):256-65.

7. Brand-Gothelf A, Parush S, Eitan Y, Admoni S, Gur E, Stein D. Sensory modulation disorder symptoms in anorexia nervosa and bulimia nervosa: A pilot The International journal of eating disorders 2016,49 (1):59-68.

8. Kinnaird E, Dandil Y, Li Z, Smith K, Pimblett C, Agbalaya R, Stewart C, Tchanturia K. Pragmatic Sensory Screening in Anorexia Nervosa and Associations with Autistic Traits. JCM 2020, 9(4).

9. Lautenbacher S, Pauls AM, Strian F, Pirke KM, Krieg JC. Pain sensitivity in anorexia nervosa and bulimia nervosa. Biol Psychiatry. 1991;29(11):1073-8.

10. Bär KJ, Berger S, Schwier C, Wutzler U, Beissner F. Insular dysfunction and descending pain inhibition in anorexia nervosa. Acta Psychiatr Scand. 2013;127(4):269-78.

11. crucianelli L, Cardi V, Treasure J, Jenkinson PM, Fotopoulou A. The perception of affective touch in anorexia nervosa. Psychiatry Res. 2016;239:72-8.

12. Goldzak-Kunik G, Friedman R, Spitz M, Sandler L, Leshem M. Intact sensory function in anorexia nervosa. Am J Clin Nutr. 2012;95(2):272-82.

13. Karavia A, Kapsali F, Gonidakis F, Koliou A, Tsigkaropoulou E, Papageorgiou C, Michopoulos I. Olfactory capacity in anorexia nervosa: correlations with set-shifting ability. Eat Weight Disord 2021.

14. Bischoff-Grethe A, Wierenga CE, Berner LA, Simmons AN, Bailer U, Paulus MP, Kaye WH. Neural hypersensitivity to pleasant touch in women remitted from anorexia nervosa. Translational 
Psychiatry. 2018;8(1):161.

15. mergen J, Keizer A, Koelkebeck K, van den Heuvel M,RC, Wagner H. Women with Anorexia Nervosa do not show altered tactile localization compared to healthy controls. Psychiatry Res. 2018;267:446-54.

16. Engel MM, Keizer A. Body representation disturbances in visual perception and affordance perception persist in eating disorder patients after completing treatment. Sci Rep. 2017;7(1):16184.

17. Verbe J, Lindberg PG, Gorwood P, Dupin L, Duriez P. Spatial hand representation in anorexia nervosa: a controlled pilot study. Sci Rep. 2021;11(1):19724.

18. Brown TA, Shott ME, Frank GKW. Body size overestimation in anorexia nervosa: Contributions of cognitive, affective, tactile and visual Psychiatry Res 2021, 297:113705.

19. Risso G, Martoni RM, Erzegovesi S, Bellodi L, Baud-Bovy G. Visuo-tactile shape perception in women with Anorexia Nervosa and healthy women with and Neuropsychologia 2020, 149:107635.

20. Engel MM, van Denderen K, Bakker A, Corcoran AW, Keizer A, Dijkerman HC. Anorexia nervosa and the size-weight illusion: No evidence of impaired visual-haptic object integration. PLOS ONE. 2020;15(8):e0237421.

21. Lock J, Le Grange D, Agras WS, Moye A, Bryson SW, Jo B. Randomized clinical trial comparing family-based treatment with adolescent-focused individual therapy for adolescents with anorexia nervosa. Arch Gen Psychiatry. 2010;67(10):1025-32.

22. Dunn W, Westman K. The sensory profile: the performance of a national sample of children without disabilities. Am J Occup Ther. 1997;51(1):25-34.

23. Brown C, Tollefson N, Dunn W, Cromwell R, Filion D. The Adult Sensory Profile: measuring patterns of sensory processing. Am J Occup Ther 2001 Am J Occup Ther. 2001;55(1):75-82.

24. Dunn W. Performance of typical children on the Sensory Profile: an item analysis. Am J Occup Ther. 1994;48(11):967-74.

25. Ito H, Hirashima T, Hagiwara T, Iwanaga R, Tani I, Yikihiro R. Standardization of the Japanese version of the sensory profile: reliability and norms Based on a community sample. Seishin Igaku. 2013;55:537-48.

26. Hirashima T, Ito H, Iwanaga R, Hagiwara T, Tani I, Yukihiro R, Tsujii M. Construct validity of the Japanese version of the Adolescent/Adult Sensory Seishinigaku. 2014;56(2):123-32.

27. Maloney MJ, McGuire J, Daniels SR, Specker B. Dieting behavior and eating attitudes in children. Pediatrics. 1989;84(3):482-9.

28. Garner DM, Olmsted MP, Bohr Y, Garfinkel PE. The eating attitudes test: psychometric features and clinical correlates. Psychol Med 1982 Psychol Med. 1982;12(4):871-8.

29. Chiba H, Nagamitsu S, Sakurai R, Mukai T, Shintou H, Koyanagi K, Yamashita Y, Kakuma T, Uchimura N, Matsuishi T. Children's Eating Attitudes Test: Children's Eating Attitudes Test: Reliability and validation in Japanese adolescents. Eating Behav. 2016;23:120-5.

30. Auyeung B, Baron-Cohen S, Wheelwright S, Allison C. The Autism Spectrum Quotient: Children's Version (AQ-Child). J Autism Dev Disord. 2008;38(7):1230-40. 
31. Wakabayashi A, Baron-Cohen S, Uchiyama T, Yoshida Y, Tojo Y, Kuroda M, Wheelwright S. The autism-spectrum quotient (AQ) children's version in Japan: A cross-cultural comparison. J Autism Dev Disord. 2007;37(3):491-500.

32. Frank GKW. Neuroimaging and eating disorders. Curr Opin Psychiatry. 2019;32(6):478-83.

33. DeGuzman M, Shott ME, Yang TT, Riederer J, Frank GKW. Association of Elevated Reward Prediction Error Response With Weight Gain in Adolescent Anorexia Nervosa. Am J Psychiatry. 2017;174(6):557-65.

34. Frank GK, Collier S, Shott ME, O'Reilly RC. Prediction error and somatosensory insula activation in women recovered from anorexia nervosa. J Psychiatry Neurosci. 2016;41(5):304-11.

35. Frank GKW, Kalina C, DeGuzman MC, Shott ME. Eye blink and reward prediction error response in anorexia nervosa. Int J Eat Disord. 2020;53(9):1544-9.

36. Sternheim LC, Wickham MI, Danner UN, Maddox TW, Filoteo VJ, Shott ME, Frank GKW. Understanding implicit and explicit learning in adolescents with and Journal of eating disorders 2021,9 (1):1-77.

37. Epstein J, Wiseman CV, Sunday SR, Klapper F, Alkalay L, Halmi KA. Neurocognitive evidence favors "top down" over "bottom up". mechanisms in the Eat Weight Disord. 2001;6(3):140-7.

38. Seidel M, Ehrlich S, Breithaupt L, Welch E, Wiklund C, Hübel C, Thornton LM, Savva A, Fundin BT, Pege J, Billger A, Abbaspour A, Schaefer M, Boehm I, Zvrskovec J, Rosager EV, Hasselbalch KC, Leppä V, Sjögren M, Nergårdh R, Feusner JD, Ghaderi A, Bulik CM. Study protocol of comprehensive risk evaluation for anorexia nervosa in twins (CREAT): a study of discordant monozygotic twins with anorexia nervosa. BMC Psychiatry. 2020;20(1):507-7.

\section{Tables}


Table 1

Demographic and background data

\begin{tabular}{|c|c|c|c|c|}
\hline & HC group $(n=63)$ & AN group $(n=17)$ & $p$ & $d, r$ \\
\hline Age (months) & $162 \pm 8$ & $164 \pm 19$ & 0.29 & $d=0.18$ \\
\hline Height (cm) & $154.1 \pm 6.4$ & $150.3 \pm 9.2$ & 0.05 & $d=0.54$ \\
\hline Weight (kg) & $45.9 \pm 6.4$ & $31.0 \pm 6.5$ & $<0.01$ & $d=2.31$ \\
\hline $\mathrm{BMI}\left(\mathrm{kg} / \mathrm{m}^{2}\right)$ & $19.3 \pm 2.1$ & $13.6 \pm 1.8$ & $<0.01$ & $d=2.76$ \\
\hline BMI-SDS & $-0.2 \pm 0.9$ & $-3.8 \pm 1.6$ & $<0.01$ & $d=3.36$ \\
\hline Duration of illness (months) & & $8 \pm 5$ & & \\
\hline ChEAT26 total score & $8(0-39)$ & $25(3-51)$ & $<0.01$ & $r=0.45$ \\
\hline Preoccupation with thinness & $1(0-13)$ & $5(0-15)$ & 0.34 & $r=0.11$ \\
\hline Food preoccupation & $0(0-6)$ & $3(0-14)$ & $<0.01$ & $r=0.47$ \\
\hline Dieting & $3(0-13)$ & $7(3-21)$ & $<0.01$ & $r=0.56$ \\
\hline Social pressure to eat & $0(0-10)$ & $6(0-11)$ & $<0.01$ & $r=0.53$ \\
\hline Purging & $0(0-2)$ & $0(0-3)$ & $<0.01$ & $r=0.43$ \\
\hline Total AQC score & $14(5-38)$ & 19(11-34) & 0.07 & $r=0.20$ \\
\hline Social skills & $4(0-10)$ & $4(0-9)$ & 1.00 & $r=0.00$ \\
\hline Attention switching & $3(0-9)$ & $4(1-8)$ & 0.06 & $r=0.21$ \\
\hline Attention to detail & $3(1-7)$ & $5(0-7)$ & 0.01 & $r=0.29$ \\
\hline Communication & $2(0-8)$ & $3(0-6)$ & 0.14 & $r=0.16$ \\
\hline Imagination & $3(0-9)$ & $3(1-6)$ & 0.98 & $r=0.00$ \\
\hline \multicolumn{5}{|c|}{$\begin{array}{l}\text { Statistics reported are means ( } \pm \text { standard deviation) for age, height, weight, BMI, BMI-SDS and } \\
\text { duration of illness. Due to nonnormal distributions, statistics reported are medians followed by the } \\
\text { range in parentheses for ChEAT26 and AQC scores. }\end{array}$} \\
\hline \multicolumn{5}{|c|}{$\begin{array}{l}\text { HC: healthy control, AN: anorexia nervosa, BMI: body mass index, SDS: standard deviation score, } \\
\text { ChEAT26: Children's Eating Attitude Test, AQC: Autism-Spectrum Quotient Children's version }\end{array}$} \\
\hline
\end{tabular}


Table 2

AASP scores

\begin{tabular}{|lllll|}
\hline & HC group (n=63) & AN group (n=17) & $p$ & $r$ \\
\hline Low Registration & $29(15-53)$ & $31(17-45)$ & 0.92 & 0.01 \\
\hline Sensation Seeking & $37(16-52)$ & $36(27-50)$ & 0.58 & 0.06 \\
\hline Sensory Sensitivity & $30(15-51)$ & $34(21-53)$ & 0.14 & 0.17 \\
\hline Sensation Avoiding & $30(15-52)$ & $32(19-46)$ & 0.30 & 0.12 \\
\hline Taste/Smell processing & $16(8-26)$ & $18(11-28)$ & 0.10 & 0.18 \\
\hline Movement processing & $16(8-26)$ & $20(10-27)$ & 0.05 & 0.22 \\
\hline Visual processing & $22(10-33)$ & $22(15-31)$ & 0.13 & 0.17 \\
\hline Touch processing & $26(13-46)$ & $31(16-42)$ & 0.09 & 0.19 \\
\hline Activity level processing & $26(10-39)$ & $24(12-29)$ & 0.08 & 0.19 \\
\hline Auditory processing & $24(11-43)$ & $25(12-37)$ & 0.76 & 0.03 \\
\hline Due to nonnormal distributions, statistics reported are medians followed by the range in parentheses. \\
\hline HC: healthy control, AN: anorexia nervosa, AASP: Adolescent/Adult Sensory Profile & & \\
\hline
\end{tabular}


Table 3

SP scores

\begin{tabular}{|c|c|c|c|c|}
\hline & $\begin{array}{l}\text { HC group } \\
(n=63)\end{array}$ & $\begin{array}{l}\text { AN group } \\
(n=17)\end{array}$ & $p$ & $r$ \\
\hline Low Registration & $17(15-36)$ & $17(15-51)$ & 1.0 & 0.00 \\
\hline Sensation Seeking & $26(26-47)$ & $28(26-37)$ & 0.09 & 0.19 \\
\hline Sensory Sensitivity & $23(20-43)$ & 23(19-38) & 0.82 & 0.03 \\
\hline Sensation Avoiding & $39(30-90)$ & $52(35-93)$ & $<0.01$ & 0.35 \\
\hline Auditory processing & $10(8-20)$ & $9(7-24)$ & 0.08 & 0.2 \\
\hline Visual processing & $10(9-22)$ & $12(9-26)$ & 0.04 & 0.23 \\
\hline Vestibular processing & $11(11-21)$ & 11(11-21) & 0.66 & 0.05 \\
\hline Touch processing & 19(18-32) & 20(18-37) & 0.14 & 0.17 \\
\hline Multisensory processing & $7(7-17)$ & $7(7-11)$ & 0.34 & 0.04 \\
\hline Oral sensory processing & $12(12-30)$ & 13(12-28) & 0.29 & 0.12 \\
\hline Sensory processing related to endurance/tone & $10(9-25)$ & $9(9-37)$ & 0.83 & 0.02 \\
\hline Modulation related to body position \& movement & $10(10-16)$ & 10(10-17) & 0.54 & 0.07 \\
\hline Modulation of movement affecting activity level & $11(7-28)$ & 13(7-23) & 0.23 & 0.13 \\
\hline $\begin{array}{l}\text { Modulation of sensory input affecting emotional } \\
\text { responses }\end{array}$ & $4(4-10)$ & $4(4-8)$ & 0.64 & 0.05 \\
\hline $\begin{array}{l}\text { Modulation of visual input affecting emotional } \\
\text { responses and activity level }\end{array}$ & $4(4-14)$ & $4(3-10)$ & 0.35 & 0.11 \\
\hline Emotional/Social responses & $22(17-65)$ & $36(21-56)$ & $<0.01$ & 0.46 \\
\hline Behavioral outcomes of sensory processing & $7(6-18)$ & $10(6-14)$ & 0.12 & 0.18 \\
\hline Items indicating thresholds for response & $3(3-5)$ & $3(3-4)$ & 0.80 & 0.03 \\
\hline \multicolumn{5}{|c|}{ Due to nonnormal distributions, statistics reported are medians followed by the range in parentheses. } \\
\hline HC: healthy control, AN: anorexia nervosa, SP: S & file & & & \\
\hline
\end{tabular}

Table 4 is not available with this version 